Personal View Article

\title{
Peaceful End of Life Theory: A Critical Analysis of Its Use to Improve Nursing Practice
}

Schadrack Ngabonziza ${ }^{1 *}, 3$, Marie Chantal Murekatete ${ }^{2}$, Gerard Nyiringango ${ }^{2}$, Sandra Marie Grace Musabwasoni ${ }^{3,1}$

${ }^{1}$ Clinical Medicine and Community Health, College of Medicine and Health Sciences, University of Rwanda

${ }^{2}$ Nursing Departemnet, School of Nursing and Midwifery, College of Medicine and Health Sciences ,University of Rwanda

${ }^{3}$ Arthur Labatt Family School of Nursing, Western University, London, Ontario, Canada

*Corresponding author: Schadrack Ngabonziza. Clinical Medicine and Community Health, College of Medicine and Health Sciences ,University of Rwanda. Email: sngabonz@uwo.ca

\begin{abstract}
Background

Provision of empathetic palliative care in agreement with patient's favorites is an indispensable attitude of healthcare providers. A Peaceful End of Life Theory was designed by Rulandand Moore (1998),to provide theoretical framework for nurses who care for patients at end stage of their life.

Methods

Chinn and Kramer theory analysis guideline was used to analyse this theory to suggest its improvement.

\section{Results}

Five major concepts and sub-concepts are identified.This theory informs the nursing profession on the relieving interventions at the end of life. It provides a guidance to supportively manage terminally ill patients in collaboration with their families.

\section{Conclusion}

This theory is important to guide nursing practice,research, and education. However, there is a lack of an instrument to measure the desired outcomes, some subconcepts do not cleary specify the nursing interventions, and it lacks the spiritual comfort to the terminally ill patients who believe in eternal life.

Rwanda J Med Health Sci 2021;4(3):412-417
\end{abstract}

Keywords: Peaceful end of life, significant others, terminally ill patients, theory analysis.

\section{Introduction}

The world's increasing aging population, malignant tumors, and other incurable diseases have made the end of life care (ELC) and a peaceful death an indispensable element of nursing care. [1] The World Health Organization (WHO) defined ELC as the interventions that expand the patients' wellbeing and their families facing problems associated with severe incurable ailments, its inhibition and alleviation of unnecessary suffering through early detection, holistic examination and pain management.[1] The ELC should provide information for emotional, physical, and spiritual needs.[2] and it depends on nurses' capacity to identify and understand the problems of dying patients.[3]
It is meaningful to patients and their families to support the dying patients physically, psychologically, and spiritually to allow them to feel peaceful with as much dignity and grace as possible.[4] However, clinical guidelines informing nurses about nursing care of patients at the last stage of chronic diseases are not available in all settings. For this sake, the Peaceful End of Life Theory (PELT) was designed by Ruland and Moore in 1998 to provide a framework for nurses to help patients make their end of life a significant experience.[5] This analysis will help to critically understand the PELT to improve its use in nursing practice, research, and education. 


\section{Method of Analysis}

This analysis is based on the six criteria of theory analysis of Chinn and Kramer (2015), which are "what is the purpose of the theory?", "what are the concepts of the theory?", "how are the concepts defined within the theory?", "what is the nature of the relationships within the theory?", "what is the structure of the theory?", and "on what assumptions does the theory build on?”.[6]

\section{Description of the Theory}

Chinn and Kramer (2015), defined theory analysis as the method of questioning the components of the theory to grasp its meaning and its usefulness and further determine if modifications are needed to improve it.[6]

\section{Purpose of theTheory.}

The PELT was developed to respond to the lack of clinical guidelines for nurses who were caring for critically ill patients in the intensive care unit (ICU) at the University hospital in Norway. Even if the nursing interventions and outcomes are specific to patients and their family members, the nursing goal of PELT is to enhance the management of near to die ill patients by providing the optimal care offered by using technology and comfortable interventions to avoid suffering.[5]

\section{Concepts and Sub-concepts of the Theory}

Chinn and Kramer (2015), define concepts as structured and related ideas within the theory. The concepts within the PELT are five and have the following sub-concepts: (1) Not being in pain: It is achieved when nurses check and administer pain relief by applying nonpharmacological and pharmacological interventions to enhance the patient's experience of not being in pain. (2) Experience of comfort: It includes prevention, monitoring, and alleviation of physical distress, relaxation, facilitating rest, contentment, and avoiding complications.(3) Experience of dignity/respect: This is enhanced by incorporating the patient and significant relatives in the management of the patient, take care of the patient with empathy, dignity, respect, and paying attention to the patient's expressed preferences, wishes, and needs.(4) Being at peace: It is all about psychological support, monitoring and responding to the patient's wishes for anti-anxiety drugs, guide the patient and relatives in practical issues, including the presence of the guardian in the management whenever needed, and inspiring trust.
(5) Closeness to significant others/persons who care: This is enhanced by the involvement of important relatives in inpatient care, attending to the relatives' worries, grief, and enquiries, and facilitating occasions to stay with close family members.[5]

\section{Definition of the Concepts within the Theory}

The definition is the meaning explicitly conveyed to the concept by the author to help others comprehend how ideas are represented and expressed in the theory.[6]

Not Being in Pain had been described as "not having any sort of pain. Pain is described as unpleasant, sensory and emotional experience which is associated with any actual or potential tissue damage",(5 p.172).

Experience of Comfort is defined as "relief from discomfort; the state of ease and peaceful contentment and whatever makes life easy or pleasurable",(5p.172).

Experience of Dignity or Respect is welldefined as "being respected and valued as a human being. Every human being likes to be respected and valued" (5 p.172).

Being at Peace comprises a feeling of tranquility, calmness, harmony, and satisfaction. It is recommended that patients should not experience any sorts of anxieties, restlessness, and fear.[5]

Closeness to Significant Others/Persons who Care is described as the moods of feeling connected to friends and families. It is facilitated by the opportunities for patients to meet and talk with their close relatives.[5,7]

\section{Nature of the Relationships within the Theory}

Relationships show how concepts are interconnected, the different forms that can establish relational statements, and how the concepts give structure to the theory.[6] In the PELT, the concepts are independent, with no relation from one to another or have to be achieved before another. However, the author has shown the vertical relationship between peaceful end of life with each concept.[5]

\section{The structure of the theory}

The diagram that illustrates aspects of the structure is provided (Fig. 1).The structure links peaceful end of life with the concepts that enhance its achievement and the concepts to their sub-concepts. The relationship in this structure move vertically from each concept to their sub-concepts (interventions). The subconcepts contribute to the enhancement of the concepts. Then, each concept contributes to the PELT independently. 


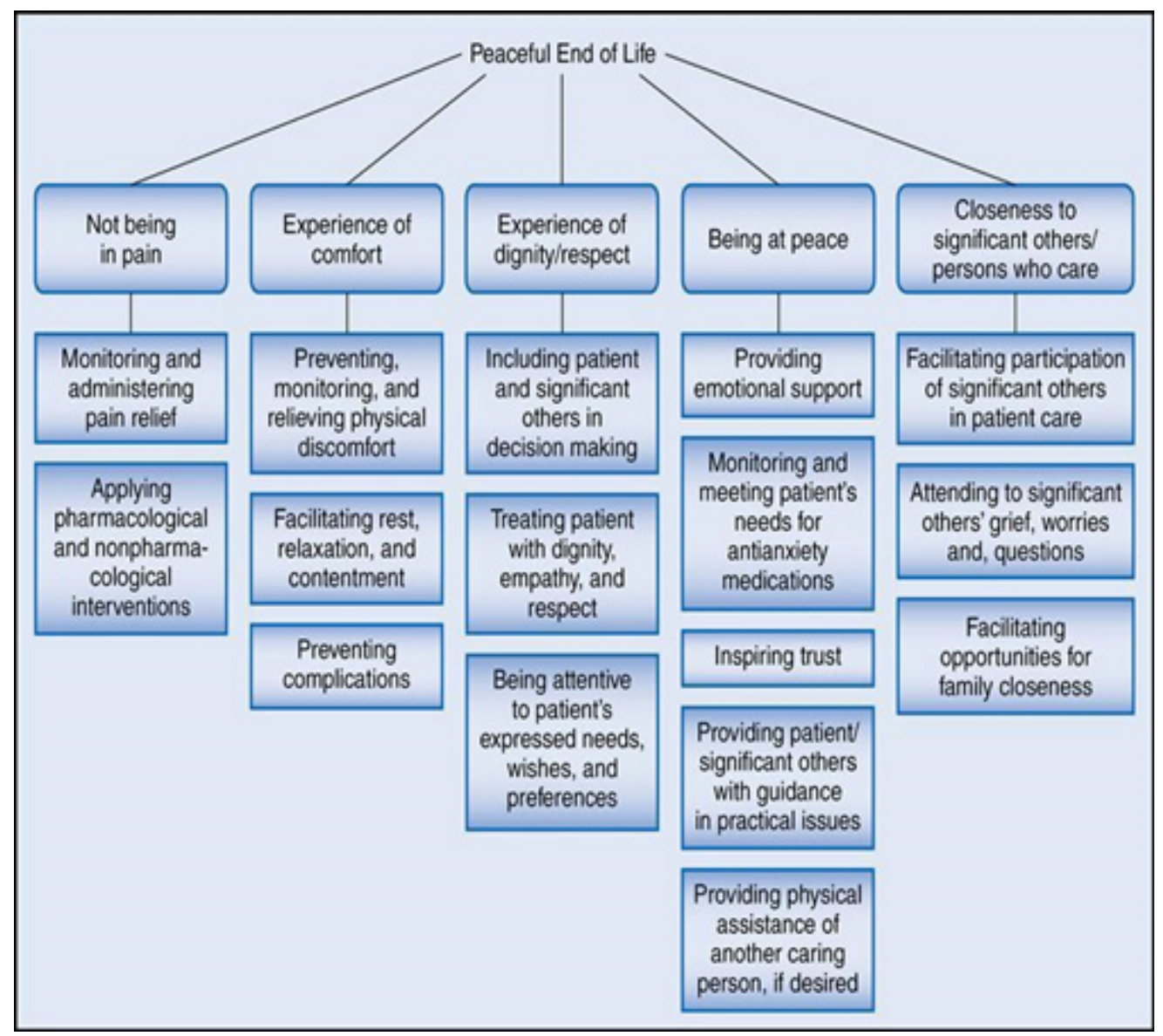

Figure 1. Structure of the theory.

From C. M. Ruland \& Moore, (1998) Theory construction based on standard of care. A proposed theory of the peaceful end of life. Nursing Outlook,46(4), 174

\section{Assumptions of the Theory}

Assumptions are the basic accepted truths that underly theoretical reasoning.[7] Ruland and Moore (1998) assumed that "a person's approach to the end of life is a highly personal experience that can be facilitated by nurses".[5] Changes Occurring over Time

Chinn and Kramer (2015), specified that a "theory is not a finalized prescription or a formula for practice; It cannot describe exactly what can be objectively observed. Instead, theory projects tentative ideas that open new perceptions and possibilities about what might be beyond the common surface of understanding the world" $(p$ 157). Ruland and Moore expressed the need for further improvement and expansion of PELT. [7] Yet, we did not find any new version of this theory showing changes that have occurred over time.

\section{Critiques of the Theory}

Chinn and Kramer(2015), asserted that "theory is grounded in assumptions, value choices, and the creative and imaginative judgment of the theorist. You may or may not share the values and views of the theorist, but your exposure to the theory and the views that it reveals can expand your thinking about your experience, your profession, and the direction of your work" (p,157). The provided critiques are our understandings and perceptions about PELT based on the criteria of chinn and Kramer (2015).

Clarity of theory describes how the theory can be unambiguously comprehended and how the concepts are presented.[6] After reading and reflecting on this PELT, we have found that the concepts within the theory are comprehensively defined without room for confusion. The sense of the concepts is maintained within the description of the theory, but the sub-concepts/ interventions are not defined. Some interventions specify what to do throughout this theory and other interventions are broad and not specific. For example, inspiring trust and providing emotional support are good components in caring for a terminally ill patient, but how can it be done?. Each nurse can understand it as he/she wants. Based on the above fact, some concepts are not clear enough to guide nursing in practice.

Simplicity refers to a small number of the concepts and their interrelationships.[6] This theory contains five concepts and each concept has many sub-concepts. There are no interrelationships between the concepts. 
The concepts are too abstract to be measured and no instrument or tool has been developed to measure accurately the outcome of interventions for the concepts. Furthermore, it is difficult for a nurse to put in action the psychological management once he / she has many patients to care for. In conclusion, this theory is complex to guide practice for nurses who care for terminally ill patients as it has many concepts and subconcepts which are also abstract and difficult to measure to detemine whether the patient experiences peace at the end of life or not.

Generality, grounded on the definition of Chinn and Kramer (2015), the generality of a theory looks at its wide range of practice and goal. A general theory is utilised in different situations and settings.[6] This PELT can be applied to various clients at the end stage of life and their families while receiving care from any department and for any disease. It is designed to carry out a peaceful death for every patient with incurable conditions. This theory has inspired many researchers in the studies conducted about the peaceful end of life as described below in its importance. Based on its broad spectrum of application, we concluded that this theory has general applicability within the nursing profession.

Accessibility, Chinn and Kramer (2015) illustrate accessibility as "the extent to which empirical indicators for the concepts can be identified and to what extent the purposes of the theory can be attained".(p.205) Based on the articles explored, the concepts of this theory have not been empirically tested to establish relationships between concepts and sub-concepts. The studies inspired by this theory were mainly looking for obstacles perceived by nurses to provide the management that enhance peace at the end of life for the patients. There is no proven evidence that these concepts and sub-concepts enhance the attainment of peaceful death for patients. In conclusion, this theory has not been empirically assessed for its accessibility.

Importance of the theory to nursing practice is determined by its usefulness in clinical setting or its clinical implications. A significant theory is effective in practice, education, and research and is valued for producing an anticipated future.[6] In practice, this theory serves as a guideline for nurses to peacefully manage the patients at the end of life and support their families in dealing with grief. It uses a family-centered approach which is nowadays important in a caring system. In education, the PELT can be used to guide students about the holistic care of patients and their families. In research, this theory has inspired Beckstrand, and Callister, (2006) in their study entitled "Providing a Good Death". The study collected suggestions from nurses to enhance end of life care but it did not measure the concepts of this theory. [8]

Beckstrand (2001), referred to the PELT to conduct a study for her doctoral dissertation entitled "National Survey of Critical Care Nurses: Perceptions of End-ofLife Care and Effect of Incentives on Survey Response Rates". This study addressed nurse encountered problems and suggestions to manage peacefully the patients at end of life.[9] In Thailand, a theoretical model to guide the nurses to promote a peaceful care at the end of life in the context of Thai Buddhism was synthesized from the PELT.[10] All the above testify that the PELT is important in the nursing profession.

Strengths of the Theory, the PELT is an important and valuable theory in the nursing profession. It provides a guideline for nurses to treat the suffering clients at end of their life to let them go with much dignity as possible.[5] It uses the familycentered approach.This approach helps terminally ill clients and their families in dealing with sorrow, loss, and anxiety related to the loss of their beloved person. Weakness of the Theory, even if this theory has its strengths, there are also some weaknesses. No instrument or tool has been yet developed to measure the concepts of this theory and its relationship. For the believers, the theorists did not consider how spiritual support inspire trust to enhance the patient's state of being at peace. For some concepts such as being at peace, the suggested interventions are broad and not specific. Many tasks need psychological care to prepare patients and families to cope with the anguish situation but this theory does not promote the use of counselors. This pushes for suggesting its improvement.

\section{Discussion}

This theory provides a theoretical framework for nurses who care for patients at the end of their life. Ruland and Moore (1998), developed this PELT to guide the nursing practice and let the patients die without avoidable suffering in collaboration with the families. [5] This PELT has been used to guide nurses in practice, research, and education.[8] In this theory, definitions of concepts are understandable as they are defined using the common terms within the nursing domain.[11] However, some of the interventions are broad and not specific. 
A PELT aims to optimize the management of terminally ill patients by use of relief measures and technology to enhance peaceful mood at end-of-life. However, during the analysis of this theory, we did not find the technology to be used. Based on the description and critique of this theory; there is no empirical evidence yet to show that this theory contributes to a peaceful end of life. We agree with the assumption of the theorists that death is personal experience and nurses in most of the healthcare settings are the right persons to care for the patients at end of life. However, it has been revealed that nurses are overwhelmed by families who do not accept their relative's poor prognosis and continue to call nurses for help when there is nothing more to do for the patient.[12] This shows that nurses alone are not enoungh to provide the holistic management for the patients at end of life and deal with the stress of their relatives.

When you look at this theory you can think that it is simple to understand and to use. But when you examine it deeply, it is complex. All concepts and some of the sub-concepts are too abstract to be measured. However, this theory is important in the nursing domain as it serves as a guidence for nurses to manage the patient at terminal stage where there are no clearly defined guidelines.

\section{Conclusion and Suggestions}

The authors of this critique acknowledge greatly the work done by the theorists to provide the theoretical framework for nurses who care for terminally ill patients. Throughout the development of this theory five major concepts were identified. Each concept is independent and is only connected to its sub-concepts. Critically, PELT is not clear enough and is complex but important to provide direction to nursing practice and research. It is general, as its scope of practice applies to all terminally ill patients. Despite its contribution of providing the theoretical framework in caring for terminally ill patients towards a peaceful death, it lacks instruments to measure the desired outcomes, and some interventions are too broad and can create confusion for nurses. In addition, the theorists did not put much emphasis on the spiritual comfort of terminally ill patients.
For refinement of the theory, the following suggestions can be considered.

1. Tools to measure outcomes need to be developed to determine the level of peace in the patients at end of life and their families.

2. To specifically provide tasks to be done by nurses to guide them in implementing the theory in their daily activities.

3. It would be better to have the counselors to accompany the patients and their families to deal with death.

4. Theorists can incorporate spiritual support for those who believe in eternal life.

5. Further researches are needed to empirically test the concepts of this theory.

\section{Conflict of interest}

The authors declare that there is no conflict of interest

\section{Authors'contribution}

SN chose the theory, analysis framework and carried out the theory analysis.

M C M ensured that questions designed by the analysis framework are appropriately examined and answered.

$\mathrm{G} N$ revised critically the PELT analysis for intellectual content.

M SG $M$ edited this work and approved the version to be published.

All authors read and approved this analysis.

This article is published open access under the Creative Commons Attribution-NonCommercial NoDerivatives (CC BYNC-ND4.0). People can copy and redistribute the article only for noncommercial purposes and as long as they give appropriate credit to the authors. They cannot distribute any modified material obtained by remixing, transforming or building upon this article. See https:// creativecommons.org/licenses/by-nc-nd/4.0/

\section{References}

1. WHO. Global atlas of palliative care at the end of life [Internet]. 2014. Available from: https://www.who.int/nmh/Global_Atlas_of_ Palliative_Care.pdf

2. Gail P.Ciccarello. Strategies to Improve End of Life Care in the Intensive Care Unit. Dimens Crit care Nurs . 2003;22:216-22. Available from: http://web.b.ebscohost. com.proxy 1.lib.uwo.ca/ehost/pdfviewer/ pdfviewer?vid=1\&sid=efc21135-9841-4184a4bd-53dd81a94013\%40pdc-v-sessmgr04

3. Berton TC. Increasing Comfort With End of Life Discussions [Internet]. University of Nevada, Las Vegas. 2015. Available from: http://digitalscholarship.unlv.edu/ thesesdissertations/2332/ 
4. Keegan L, Drick CA. End of life: Nursing solutions for death with dignity. New York: Springer Publishing Company; 2010.

5. Ruland CM, Moore SM.Theory construction basedonstandards of care:aproposed theory of the peaceful end oflife. Nurs Outlook[Internet]. 1998;46:169-75. Available from: http:// ac.els-cdn.com/S0029655498900690/1 s2.0-S0029655498900690-main.pdf? tid=b17be276-f769-11e4-81fb-00000aacb3 5e\&acdnat=1431299585_30b50e9dfe74366 89eb4f12163a6fc06

6. Chinn P., Kramer M. Knowledge development in nursing:Theory and process. 9 th Editiion. Elsevier Canada; 2015.

7. Alligood M, Tomey AM. Nursing Theorists and Their Work. 7th edition. Alexopoulos $Y$, editor. Elsevier Mosby. MOSBY Elsevier; 2009.

8. Beckstrand R, Callister LC, Kirchhoff KT. Providing A"Good Death": Critical Care Nurses'Suggestions for Improving End of Life Care. Amercan $J$ Crit Care [Internet]. 2006;15:8. Available from: https:// search-proquest-com.proxy1.lib.uwo.ca/ docview/227910189/fulltextPDF
9. Beckstrand RL. National survey of critical care nurses'perceptions of end of life care and effect of incentives on survey response rates [Internet]. University of Utah; 2001. Available from: https://collections.lib.utah. edu/details?id=191932

10. Kongsuwan W, Touhy T. Promoting Peaceful Death for Thai Buddhists. Holist Nurs Pract.2009;23:289-96

11.Alligood MR. Nursing Theorists and Their Work. 8th Edition. Elsevier Mosby. Mosby; 2014.

12. Lamoreaux N. Critical Care Nurses , Perceptions of End of Life Care : Comparative 17-year Data [Internet]. Brigham Young University; 2016. Available from: http:// scholarsarchive.byu.edu/etd 